\title{
Auto Detection of Number Plate of Person without Helmet
}

\author{
${ }^{1}$ Shanmugaraj.S, ${ }^{2}$ Thiyagu. D, ${ }^{3}$ Vetriselvam. M, ${ }^{4}$ Narendrakumar. A. R \\ Department of Computer Science and Engineering \\ University College of Engineering - Thirukkuvalai - 610204. \\ Email ID: selvamvetri27@gmail.com
}

\begin{abstract}
Automated Number Plate Recognition organization would greatly enhance the ability of police to detect criminal commotion that involves the use of motor vehicles. Automatic video investigation from traffic surveillance cameras is a fast-emerging field based on workstation vision techniques. It is a key technology to public safety, intelligent transport system (ITS) and for efficient administration of traffic without wearing helmet. In recent years, there has been an increased scope for involuntary analysis of traffic activity. It defines video analytics as computer-vision-based supervision algorithms and systems to extract contextual information from video. In traffic circumstancesnumeroussupervise objectives can be continue by the application of computer vision and pattern gratitude techniques, including the recognition of traffic violations (e.g., illegal turns and one-way streets) and the classification of road users (e.g., vehicles, motorbikes, and pedestrians). Currently most reliable approach is through the acknowledgment of number plates, i.e., automatic number plate recognition (ANPR).
\end{abstract}

Keywords-Automated Number Plate Recognition, Video Analysis, Intelligent Transport System, Computer-Vision-based Surveillance Algorithms, Helmet Detection.

\section{INTRODUCTION}

The increasing increase of contemporary urban and national road network over the last three decades emerge the need of efficient observe and administration of road traffic. Conventional techniques for traffic dimensions, such as inductive loops, sensors or EM microwave detectors, suffer from serious inadequacy, expensive to install, they demand traffic disruption during putting in place or maintenance, they are colossal and they are unable to detect slow or impermanent stop vehicles. On the contrary, systems that are based on video are easy to install, use the breathing infrastructure of traffic surveillance. Furthermore, they can be easily improvement and they offer the flexibility to redesign the arrangement and its functionality by simply varying the system algorithms. Those systems allow quantity of vehicle's speed, together with the number of vehicles, classification of vehicles, and the classification of traffic incident (such as misfortune or heavy overcrowding). There is a wide multiplicity of systems based on video and image dispensation employing different methodologies to detect means of transport and objects.
Video acquisition Vehicle detection and tracking

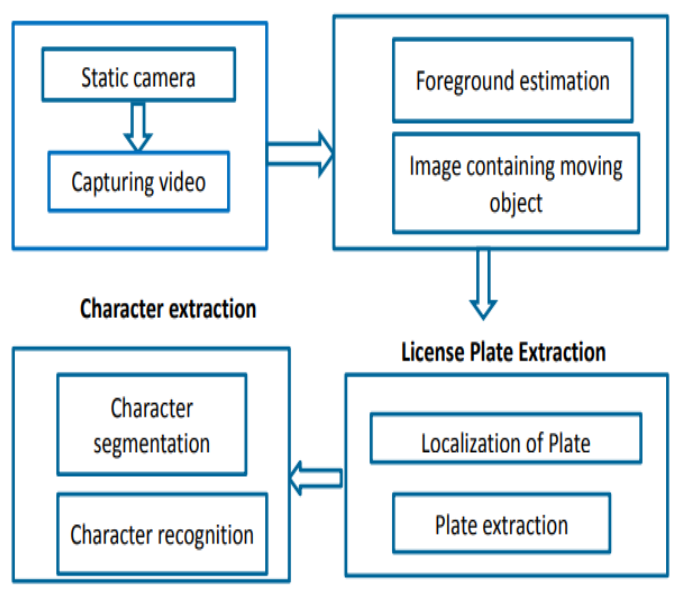

Figure 1.1 Overview of Proposed System

For most traffic observation systems there are three major stages which are used for assessment of desired traffic parameters i.e. vehicle detection, tracking, and arrangement. For detection of vehicles, most of the methods [6]-[12] assume that the camera is static and then required vehicles can be detected by image differencing. Tracking is a very significant issue in computer vision. Recently, there is a deep interest in observation applications. The main purpose of tracking in computer vision is to distinguish and locate a prototype in a series of chronological frames. Many applications are based on pathway such as video dispensation, security, surveillance and automatic measures. Then, different trail scheme are designed to track each motor 
vehicle. After that, several vehicle features like shape, measurement lengthwise, width, texture, Number etc., are extracted for vehicle categorization. Every visual surveillance system start with detect moving object in video stream.

\subsection{PROBLEM STATEMENT}

To design and develop a real-time detection, tracking and numeral plate recognition system that will work economically under the circumstances of slow moving substance and the objects that are merged into the environment due to a temporary stop and becoming forefront again, adaptive to different traffic surroundings conditions, robustness against progressive or sudden enlightenment changes, Occlusions, identification time of the system should be as short as possible. The arrangement should detect all the types of means of transport, be familiar with all the Number plates of the country and should also be opposed to to any kinds of turbulence, which may occur in images and automatic plate damages which may appear in reality without wearing head covering.

\section{LITERATURE SURVEY}

Foreground inference and segmentation is the first stage of several traffic observation systems. The foreground regions are marked (e.g., mask image) for dispensation in the subsequent steps. There are two main dissimilar approaches to estimate the forefront, which both use strong assumptions to comply with the abovementioned definition. First, anenvironment model of some kind can be used to accumulate in sequence about the scene environment of a video sequence. The model is then compare to the current frame to identify dissimilarity (or "motion"), provided that the camera is stationary. This concept lends itself well for workstationperformance but leads to struggle with slowmoving traffic. Any car should be well thought-out foreground, but motionless objects are missed due to the lack of motion.[18] $\mathrm{Hu}$ et al. [1] categorized movement detection into three major programme of method as frame differencing, optical flow, and surroundings subtraction.

Frame differencing [4] is a pixel-wise differencing stuck between two or three consecutive frames in an image succession to detect regions analogous to moving object such as human and vehicles. The threshold purpose determines change and it depends on the speed of object movement If the speed of the object changes significantly, then it's difficult to uphold the quality of segmentation. The inter-frame differencing approach detects parts of heartrending objects by comparing two consecutive frames. But, it can identify only dissimilarity in the background and, for that reason; it become aware of only parts of a vehicle covering the environment in the previous frame. Despite some ornamental techniques [4] this approach cannot adequately deal with realistic traffic state of affairs where vehicles might stay behind still for a long time.

\section{Optical Flow}

To detect moving region in an image, optical flow [5] uses flow vectors of the moving objects over time. It is used for motion-based segmentation and pathwaysubmission. It is a dense field of displacement vectors which defines the transformation of each pixel region. Optical flow is best suited in presence of camera movement, but however most flow calculation methods are computationally complex and responsive to noise.

\section{Background subtraction}

The backdrop subtraction [6], [7], [8], [9], [10], and [11] is the most popular and widespread approach for motion uncovering. In this method the current image is subtracted from a reference environment image, which is upgraded during a period of time. The elimination leaves only nonstationary or new objects, which consist of whole outline region of an object. This approach is simple and computationally reasonably priced for real-time systems, but is extremely sensitive to dynamic scene changes from lightning and unconnected event etc. Therefore it is highly dependent on a good backdrop maintenance model.

\section{III.OVERVIEW OF THE PROPOSED SYSTEM}

A typical inspection system consists of a transfer camera network, which progression captured travel video on-site and transmits the extracted restriction in real time. Here our focal point is on the study of algorithmic fraction of such a system. In this thesis, we nearby full-featured vehicle detection, tracking and Number plate acknowledgment system structure, particularly designed to work on video recording.

- Video Acquisition

- Vehicle detection and tracking

- Number plate extraction

- Character recognition unit

- Helmet Detection

- Sobel Edge Detection

This system is used to detect, be familiar with and track vehicle from incoming video frames in dynamic scenes then take out the Number plate from it as shown in Figure 1.1. It has found numerous application as wide as possible such as: right of entry control in security sensitive areas, securities for community and important buildings, detection of military target area, traffic surveillance in cities and highways, uncovering of anomalies behaviour, traffic control organization for recognize vehicles that perpetrate 
traffic violation, such as reside in lanes reserved for public transport, contravention speed limits, crossing red light, entering restricted area without authorization; and among many other application. The system is designed for real time videos where a camera is used for continuous video recording of videos. The view of camera or the area enclosed by camera is fixed between entry zones and exit zone. Each frame is incessantly processed to check the presence of a motor vehicle. A defined connectedconstituent area is taken as threshold; if the detected area is above that threshold value then it will be documented as a motor vehicle and will be tracked. A distance is defined between the vehicle and the camera and when the motor vehicle comes within that range i.e vehicle's connected component area is highest, these frames of video are passed to Number plate recognition algorithm. After that acknowledgment of character takes place and data is stored and measure up to with data base.

This Proposed System encompasses mainly the following modules:

Video Acquisition - In this module video are taken by the static camera positioned at traffic scenario. Camera arrangement has the ability to broadcast images in real time to a central operational centre. The dispensation of the images can be carried out on-site saving expensive network bandwidth as it transmit only the outcome of the calculations. The whole procedure can also be perform either in real time video streaming from an operational centre or in previously stored video material.

Vehicle Detection And Tracking - In vehicle discovery we have replicated various background subtraction techniques available in the literature. The backdrop subtraction technique should overcome the trouble of varying illumination condition, background clutter, camouflage and silhouette. Motion segmentation of forefrontentity has been done in real instance. It's hard to get this entire predicament solved in one background taking away technique. So the idea was to simulate and evaluate their presentation on a variety of video data taken in different situation.

Numeral Plate mining - numeral plates are first situated in current frame then they are extract using various available practice in the literature based on Hough Transform method, Template indistinguishableprocedure, Region budding algorithm, Histogram Approach and Edge Detection Approach.

Character Extraction - Images of the extract plates are the input to this module. Here first Number plate representation is cropped in lines, and then typescript are segmented and documented.
Helmet Detection: From the previous step, understand the face of the person is not detect we move into helmet detection. In that we use Circle Hough renovate. The circle Hough Transform (CHT) is a basic method used in Digital Image Processing, for become aware of circular objects in a digital image. It is a feature extraction technique for become aware of circles. It is a specialization of Hough Transform. The purpose of the technique is to find circles in defective image inputs.

Sobel Edge Detection: The circle picture of the helmet is detected using sobel edge detection accurately. Edge discovery is a fundamental tool in the image processing predominantly in the areas of feature discovery and feature extraction which aim at identify points a digital image at which image brightness changes sharply or more formally has put an end to. The result of applying an edge detector to an image may lead to a set of associated curves that point towards the boundary of objects. The sobel operator performs a 2D spatial gradient dimension on an image.

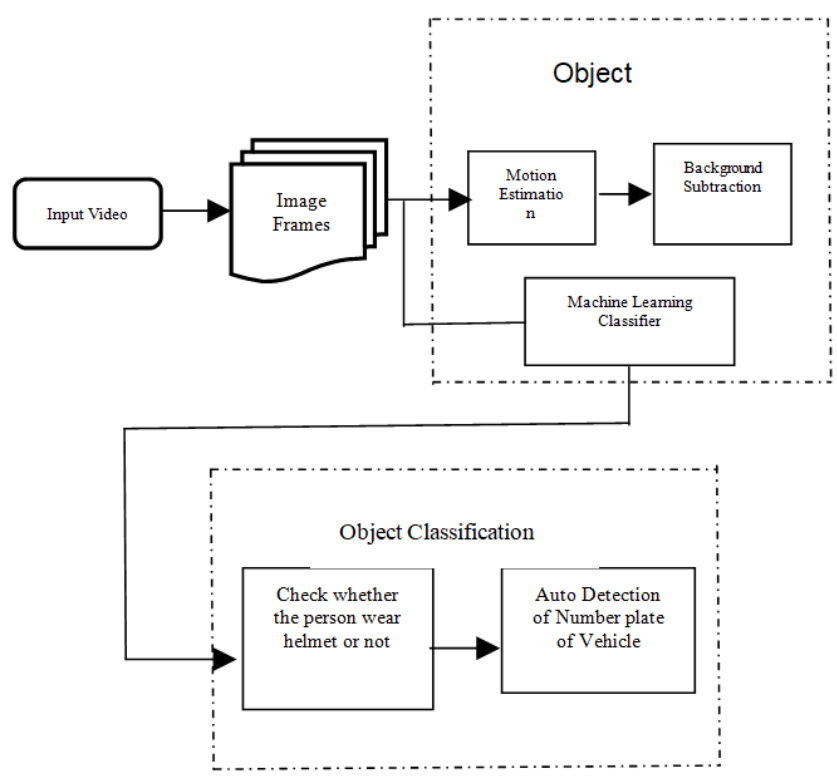

Figure 1.2 System Architecture

\section{CONCLUSION}

The proposed work urbanized a smart helmet based system which was productively able to detect whether the rider as worn the helmet or not. The main cause of these wounded is people riding two wheelers under the influence of alcohol results and infringement of traffic rules which later on results in serious accidents. The main aim of this study is to recommend and develop a system for automatic discovery of helmets on public roads. The risk of death is 2.5 times more among riders not wearing a helmet measure 
up to with those wearing a helmet. The impacts of this accident are more hazardous when the driver involves in a high speed misfortune without wearing helmet. It proposes a frame work for real time recognition of traffic rule violators who ride bike without using helmet the projected work will also assist traffic police for detect such violators in all environment circumstance and it also detect the number plate of the violators and report to the authority.

In future work, we aim to focus on night inspection and to improve the existing algorithms reported in invented story. However, the other segments of our not compulsory system should be improved, focusing on the occlusion handling, vehicle matching course of action and also focus on improving the accuracy measure for temperament recognition by using the concept of neural network for recognising all font type of a temperament by using back dissemination algorithm.

\section{REFERENCES}

[1] Hu, Weiming, Tieniu Tan, Liang Wang, and Steve Maybank. "A survey on visual surveillance of object motion and behaviors." Systems, Man, and Cybernetics, Part C: Applications and Reviews, IEEE Transactions on 34, no. 3 (2004): 334-352.

[2] Buch, Norbert, Sergio A. Velastin, and James Orwell. "A review of computer vision techniques for the analysis of urban traffic." Intelligent Transportation Systems, IEEE Transactions on 12, no. 3 (2011): 920-939.

[3] Zhu, Zhongjie, and Yuer Wang. "A hybrid algorithm for automatic segmentation of slowly moving objects." AEU-International Journal of Electronics and Communications 66, no. 3 (2012): 249-254.

[4] Lipton, Alan J., Hironobu Fujiyoshi, and Raju S. Patil. "Moving target classification and tracking from real-time video." In Applications of Computer Vision, 1998.WACV'98.Proceedings., Fourth IEEE Workshop on, pp. 8-14. IEEE, 1998

[5] Barron, John L., David J. Fleet, and S. S. Beauchemin. "Performance of optical flow techniques." International journal of computer vision 12, no. 1 (1994): 43-77.

[6] Barnich, Olivier, and Marc Van Droogenbroeck. "ViBe: A universal background subtraction algorithm for video sequences." Image Processing, IEEE Transactions on 20, no. 6 (2011): 1709- 1724.

[7] Elgammal, Ahmed, David Harwood, and Larry Davis. "Non-parametric model for background subtraction." In Computer Vision-ECCV 2000, pp. 751-767. Springer Berlin Heidelberg, 2000.

[8] Toyama, Kentaro, John Krumm, Barry Brumitt, and Brian Meyers. "Wallflower: Principles and practice of background maintenance." In Computer Vision, 1999. The Proceedings of the Seventh IEEE International Conference on, vol. 1, pp. 255-261. IEEE, 1999.

[9] Panda, Deepak Kumar. "Motion detection, object classification and tracking for visual surveillance application." PhD diss., 2012.
[10] Haritaoglu, Ismail, David Harwood, and Larry S. Davis. "W4: real-time surveillance of people and their activities." Pattern Analysis and Machine Intelligence, IEEE Transactions on 22, no. 8 (2000): 809-830.

[11] Antoine Manzanera and Julien C. Richefeu. A new motion detection algorithm based on Sigma. -Delta. background estimation. Pattern Recognition Letters, 28(3):320-328, February 2007.

[12] Manuel Vargas. et al."An Enhanced Background Estimation Algorithm for Vehicle Detection in Urban Traffic Scenes", IEEE Transactions On Vehicular Technology, Vol. 59, No. 8, October2010.

[13] Stauffer, C., \&Grimson, W. (1999). Adaptive background mixture models for real-time tracking.Computer Vision Pattern Recognition, 246-252.

[14] M. Harville, "A framework for high-level feedback to adaptive, per-pixel, mixture-of-Gaussian background models," in Proc. Eur. Conf. Comput. Vis., May 2002, vol. 3, pp. 543-560. 